Bull. Austral. Math. Soc.

VOL. 76 (2007) [69-92]

\title{
CHARACTERISATION OF EMBEDDINGS IN LORENTZ SPACES
}

\author{
A. Gogatishvili, M. Johansson, C.A. Okpoti and L.-E. Persson
}

Some new integral conditions characterising the embedding $\Lambda^{p}(v) \hookrightarrow \Gamma^{q}(w)$, $0<p, q \leqslant \infty$ are presented, including proofs also for the cases (i) $p=\infty, 0<q<\infty$, (ii) $q=\infty, 1<p<\infty$ and (iii) $p=q=\infty$. Only one condition is necessary for each case which means that our conditions are different from and simpler than other corresponding conditions in the literature. We even prove our results in a more general frame namely when the space $\Gamma^{q}(w)$ is replaced by the more general space $\Gamma_{u}^{q}(w)$. In our proof we use a technique of discretisation and anti-discretisation developed by $A$. Gogatishvili and L. Pick, where they considered the opposite embedding.

\section{INTRODUCTION}

Let $(\mathcal{R}, \mu)$ be a totally $\sigma$-finite measure space with a non-atomic measure $\mu$, and let $\mathcal{M}(\mathcal{R}, \mu)$ be the set of all extended complex-valued $\mu$-measurable functions on $\mathcal{R}$. For $f \in \mathcal{M}(\mathcal{R}, \mu)$, let

$$
f_{*}(t)=\mu(\{x \in \mathcal{R} ;|f(x)|>t\}), t \in(0, \infty),
$$

be a distribution function of $f$. The non-increasing rearrangement $f^{*}$ of $f$ is defined by

$$
f^{*}(t)=\inf \left\{x>0 ; f_{*}(x) \leqslant t\right\}, t \in(0, \infty) \text {. }
$$

We shall assume that $\mu(\mathcal{R})=\infty$. Everywhere in this paper, we assume that $u, v$, and $w$ are weights, that is, locally integrable non-negative functions on $(0, \infty)$ and we denote

$$
V(s)=\int_{0}^{s} v(t) d t \text { and } U(s)=\int_{0}^{s} u(t) d t .
$$

We assume that $u$ is such that $U(t)>0$ for every $t \in(0, \infty)$ and denote

$$
f_{u}^{* *}(t)=\frac{1}{U(t)} \int_{0}^{t} f^{*}(s) u(s) d s .
$$

Received 27th November, 2006

The research of the first author was partly supported by the grant 201/05/2033 of the Grant Agency of the Czech Republic and by the Institutional Research plan no. AV0Z10190503 of the Academy of Sciences of the Czech Republic (ASCR). The third author is grateful to the Government of Ghana for finanical support and the Department of Mathematics, Luleå University of Technology, Luleà, Sweden for providing facilities to do this research.

Copyright Clearance Centre, Inc. Serial-fee code: 0004-9727/07 \$A2.00+0.00. 
Definition 1.1: Let $p \in(0, \infty)$ and let $u$ and $v$ be weight functions. Then we define the following four spaces (of Lorentz and Marcinkiewicz type):

$$
\begin{aligned}
\Lambda^{p}(v) & =\left\{f \in \mathcal{M}(\mathcal{R}, \mu) ;\|f\|_{\Lambda^{p}(v)}=\left(\int_{0}^{\infty} f^{* p}(t) v(t) d t\right)^{1 / p}<\infty\right\} \\
\Lambda^{\infty}(v) & =\left\{f \in \mathcal{M}(\mathcal{R}, \mu) ;\|f\|_{\Lambda^{\infty}(v)}=\underset{t \in(0, \infty)}{\operatorname{ess} \sup } f^{*}(t) v(t)<\infty\right\} ; \\
\Gamma_{u}^{p}(v) & =\left\{f \in \mathcal{M}(\mathcal{R}, \mu) ;\|f\|_{\Gamma_{u}^{p}(v)}=\left(\int_{0}^{\infty} f_{u}^{* * p}(t) v(t) d t\right)^{1 / p}<\infty\right\} \\
\Gamma_{u}^{\infty}(v) & =\left\{f \in \mathcal{M}(\mathcal{R}, \mu) ;\|f\|_{\Gamma_{u}^{\infty}(v)}=\operatorname{ess~sup~}_{t \in(0, \infty)} f_{u}^{* *}(t) v(t)<\infty\right\} .
\end{aligned}
$$

When $u \equiv 1$ (hence $U(t)=t$ ), we shall omit the subscript $u$. We thus write, in such a case $f^{* *}, \Gamma^{p}(v)$ and $\Gamma^{\infty}(v)$ in place of $f_{u}^{* *}, \Gamma_{u}^{p}(v)$ and $\Gamma_{u}^{\infty}(v)$, respectively. The spaces $\Lambda^{p}(v)$ and $\Gamma^{p}(v)$ are called classical Lorentz spaces. The spaces $\Lambda^{p}(v)$ were introduced by Lorentz in 1951 in [13]. The spaces $\Gamma^{p}(v)$ were first used by Sawyer in [15] and the weak classical Lorentz spaces were introduced by Carro and Soria in [4]. Weak Lorentz spaces were further investigated in $[3,5,6,7]$. For definitions and a detailed study of rearrangement-invariant Banach function spaces see for example, [2].

During the last two decades, many authors have spent enormous efforts in order to find necessary and sufficient conditions on parameters $p, q \in[0, \infty]$ and weights $u, w$ such that the embeddings $\Lambda^{p}(v) \hookrightarrow \Gamma^{q}(w)$ hold. Such embeddings proved to be indispensable in several important areas in analysis, including the theory of interpolation and modern study of Sobolev spaces. This research brought plenty of deep results, see in for example, $[1,4,5,6,8,9,10,11,12,15,16,17,18,19,20,21]$. A summary of results of embeddings of classical Lorentz spaces known by the end the of 1990's, as well as some references, can be found in [7]. In this survey paper there were some cases of parameters which were not known. In the paper [11] all cases have been established, but the resulting characterisation were expressed in a difficult way, not very satisfactory from a practical point of view.

Our main goal in this paper is to give some new necessary and sufficient conditions on the weight functions $u, v$ and $w$ such that the inequality

$$
\|f\|_{\Gamma_{u}^{q}}(w) \leqslant C\|f\|_{\Lambda^{p}}(v)
$$

holds for every $f \in \mathcal{M}(\mathcal{R}, \mu)$.

Our approach is based on discretisation and anti-discretisation methods developed in $[9,10]$. Let as outline the structure of the paper. In Section 2 we give some preliminaries including some definitions and basic facts concerning discretisation and antidisctertisation from the papers $[9,10]$. In Section 3 we present the main results and finally in Section 4 we give the proofs. 
Throughout this paper $a \lesssim b,(b \gtrsim a)$, means that $a \leqslant \lambda b$, where $\lambda>0$ depends on inessential parameters. If $b \lesssim a \lesssim b$, then we write $a \approx b$.

\section{Preliminaries}

Let us now recall some definitions and basic facts concerning discretisation and antidiscretisation which can be found in $[9,10]$.

DEFINITION 2.1: Let $\left\{a_{k}\right\}$ be a sequence of positive real numbers. We say that $\left\{a_{k}\right\}$ is strongly increasing or strongly decreasing and write $a_{k} \uparrow \uparrow$ or $a_{k} \downarrow \downarrow$ when

$$
\inf _{k \in \mathbf{Z}} \frac{a_{k+1}}{a_{k}}>1 \text { or } \sup _{k \in \mathbf{Z}} \frac{a_{k+1}}{a_{k}}<1,
$$

respectively.

Definition 2.2: Let $U$ be a continuous strictly increasing function on $[0, \infty)$ such that $U(0)=0$ and $\lim _{t \rightarrow \infty} U(t)=\infty$. Then we say that $U$ is admissible.

Let $U$ be an admissible function. We say that a function $\varphi$ is $U$-quasiconcave if $\varphi$ is equivalent to an increasing function on $[0, \infty)$ and $\varphi / U$ is equivalent to a decreasing function on $(0, \infty)$. We say that $U$-quasiconcave function $\varphi$ is non-degenerate if

$$
\lim _{t \rightarrow 0+} \varphi(t)=\lim _{t \rightarrow \infty} \frac{1}{\varphi(t)}=\lim _{t \rightarrow \infty} \frac{\varphi(t)}{U(t)}=\lim _{t \rightarrow 0+} \frac{U(t)}{\varphi(t)}=0 .
$$

The family of non-degenerate $U$-quasiconcave functions will be denoted by $\Omega_{U}$. We say that $\varphi$ is quasiconcave when $\varphi \in \Omega_{U}$ with $U(t)=t$.

Definition 2.3: Assume that $U$ is admissible and $\varphi \in \Omega_{U}$. We say that $\left\{x_{k}\right\}_{k \in Z}$ is a discretising sequence for $\varphi$ with respect to $U$ if

(i) $x_{0}=1$ and $U\left(x_{k}\right) \pi$;

(ii) $\varphi\left(x_{k}\right) \Uparrow$ and $\left(\varphi\left(x_{k}\right) /\left(U\left(x_{k}\right)\right) \downarrow\right.$;

(iii) there is a decomposition $\mathbb{Z}=\mathbb{Z}_{1} \cup \mathbb{Z}_{2}$ such that $\mathbb{Z}_{1} \cap \mathbb{Z}_{2}=\emptyset$ and for every $t \in\left[x_{k}, x_{k+1}\right]$

$$
\begin{aligned}
& \varphi\left(x_{k}\right) \approx \varphi(t) \text { if } k \in \mathbb{Z}_{1}, \\
& \frac{\varphi\left(x_{k}\right)}{U\left(x_{k}\right)} \approx \frac{\varphi(t)}{U(t)} \text { if } k \in \mathbb{Z}_{2} .
\end{aligned}
$$

Let us recall [9, Lemma 2.7] that if $\varphi \in \Omega_{U}$, then there always exists a discretising sequence for $\varphi$ with respect to $U$.

Definition 2.4: Let $U$ be an admissible function and let $\nu$ be a non-negative Borel measure on $[0, \infty)$. We say that the function $\varphi$ defined by

$$
\varphi(t)=U(t) \int_{[0, \infty)} \frac{d \nu(s)}{U(s)+U(t)}, \quad t \in(0, \infty)
$$


is the fundamental function of the measure $\nu$ with respect to $U$. We shall also say that $\nu$ is a representation measure of $\varphi$ with respect to $U$.

We say that $\nu$ is non-degenerate if the following conditions are satisfied for every $t \in(0, \infty)$ :

$$
\int_{[0, \infty)} \frac{d \nu(s)}{U(s)+U(t)}<\infty, \quad t \in(0, \infty), \quad \int_{[0,1]} \frac{d \nu(s)}{U(s)}=\int_{[1, \infty)} d \nu(s)=\infty .
$$

We recall from $[9$, Remark 2.10] that

$$
\varphi(t) \approx \int_{[0, t]} d \nu(s)+U(t)^{q} \int_{[t, \infty)} U(s)^{-q} d \nu(s), \quad t \in(0, \infty) .
$$

We shall further assume that $\nu$ is non-degenerate.

LEMMA 2.1. Let $v$ be a weight function. Then

$$
\Lambda^{\infty}(v)=\Lambda^{\infty}(\underset{0<s<t}{\operatorname{ess} \sup } v(s))
$$

with identical norms.

The proof follows from the following simple observation: If $F$ and $G$ are non-negative functions on $(0, \infty)$ and $F$ is non-increasing, then

$$
\underset{0<t<\infty}{\operatorname{ess} \sup } F(t) G(t)=\underset{0<t<\infty}{\operatorname{ess} \sup } F(t) \underset{0<s<t}{\operatorname{ess} \sup } G(s) \text {. }
$$

LEMMA 2.2. ([10, Lemma 1.5]) Let $u, v$ be weights and let $\varphi$ be defined by

$$
\varphi(t)=\underset{s \in(0, t)}{\operatorname{esssup}} U(s) \underset{\tau \in(s, \infty)}{\operatorname{essup} \sup } \frac{v(\tau)}{U(\tau)}, \quad t \in(0, \infty)
$$

Then $\varphi$ is the least $U$-quasiconcave majorant of $v$ and

$$
\Gamma_{u}^{\infty}(v)=\Gamma_{u}^{\infty}(\varphi)
$$

with identical norms. Further, for $t \in(0, \infty)$;

$$
\begin{gathered}
\varphi(t)=\underset{\tau \in(0, \infty)}{\operatorname{ess} \sup } v(\tau) \\
\min \left\{1, \frac{U(t)}{U(\tau)}\right\}=U(t) \underset{s \in(t, \infty)}{\operatorname{ess} \sup } \frac{1}{U(s)} \underset{\tau \in(0, s)}{\operatorname{ess} \sup } v(\tau) \\
\varphi(t) \approx \underset{s \in(0, \infty)}{\operatorname{ess} \sup } \frac{v(s) U(t)}{U(s)+U(t)}
\end{gathered}
$$

TheOREM 2.1. ([9, Theorem 2.11]) Let $p, q, r \in(0, \infty)$. Assume that $U$ is an admissible function, let $\nu$ be a non-negative non-degenerate Borel measure on $[0, \infty)$ and let $\varphi$ be the fundamental function of $\nu$ with respect to $U^{q}$. Moreover, let $\sigma \in \Omega_{U}$ and let $\left\{x_{k}\right\}$ be a discretising sequence for $\varphi$ with respect to $U^{q}$. Then

$$
\int_{[0, \infty)} \frac{\varphi(t)^{(r / q)-1}}{\sigma(t)^{r / p}} d \nu(t) \approx \sum_{k \in \mathbf{Z}} \frac{\varphi\left(x_{k}\right)^{r / q}}{\sigma\left(x_{k}\right)^{r / p}}
$$


LEMMA 2.3. ([9, Corollary 2.13]) Let $q \in(0, \infty)$, let $U$ be an admissible function, let $f \in \Omega_{U}$, let $\nu$ be a positive non-degenerate Borel measure on $[0, \infty)$ and let $\varphi$ be the fundamental function of $\nu$ with respect to $U^{q}$. Moreover, assume that $\left\{x_{k}\right\}$ is a discretising sequence for $\varphi$ with respect to $U^{q}$. Then

$$
\left(\int_{[0, \infty)}\left(\frac{f(t)}{U(t)}\right)^{q} d \nu(t)\right)^{1 / q} \approx\left(\sum_{k \in Z}\left(\frac{f\left(x_{k}\right)}{U\left(x_{k}\right)}\right)^{q} \varphi\left(x_{k}\right)\right)^{1 / q}
$$

LEMмA 2.4. ([9, Lemma 3.5]) Let $U$ be an admissible function, let $f, \varphi \in \Omega_{U}$ and let $\left\{x_{k}\right\}$ be a discretising sequence for $\varphi$ with respect to $U$. Then

$$
\underset{0<t<\infty}{\operatorname{ess} \sup } \frac{f(t)}{U(t)} \varphi(t) \approx \sup _{k \in \mathbf{Z}} \frac{f\left(x_{k}\right)}{U\left(x_{k}\right)} \varphi\left(x_{k}\right)
$$

By using Lemma 3.1 and Lemma 3.2 from [9] we get following

LEMMA 2.5. If $\tau_{k} \Downarrow$, then, for any $q>0$,

$$
\begin{aligned}
& \sum_{k \in \mathbf{Z}}\left(\int_{0}^{x_{k}} h\right)^{q} \tau_{k} \approx \sum_{k \in \mathbf{Z}}\left(\int_{x_{k-1}}^{x_{k}} h\right)^{q} \tau_{k} \\
& \sup _{k \in \mathbf{Z}}\left(\int_{0}^{x_{k}} h\right)^{q} \tau_{k} \approx \sup _{k \in \mathbf{Z}}\left(\int_{x_{k-1}}^{x_{k}} h\right)^{q} \tau_{k} \\
& \sum_{k \in \mathbf{Z}}\left(\int_{x_{k}}^{\infty} h\right)^{q} \tau_{k}^{-1} \approx \sum_{k \in \mathbf{Z}}\left(\int_{x_{k}}^{x_{k+1}} h\right)^{q} \tau_{k}^{-1}
\end{aligned}
$$

and

$$
\sup _{k \in \mathbf{Z}}\left(\int_{x_{k}}^{\infty} h\right)^{q} \tau_{k}^{-1} \approx \sup _{k \in Z}\left(\int_{x_{k}}^{x_{k+1}} h\right)^{q} \tau_{k}^{-1}
$$

LEMma 2.6. ([9, Lemma 3.6]) Let $q \in(0, \infty)$, let $U$ be an admissible function, let $\nu$ be a positive non-degenerate Borel measure on $[0, \infty)$ and let $\varphi$ be the fundamental function of $\nu$ with respect to $U^{q}$. Assume that $\left\{x_{k}\right\}$ is a discretising sequence for $\varphi$ with respect to $U^{q}$. Then

$$
\begin{aligned}
\int_{0}^{\infty}\left(\int_{0}^{\infty}\right. & \left.\frac{|f(t)| d t}{U(t)+U(x)}\right)^{q} d \nu(x) \\
& \approx \sum_{k \in \mathbf{Z}}\left(\int_{0}^{\infty} \frac{|f(t)| d t}{U(t)+U\left(x_{k}\right)}\right)^{q} \varphi\left(x_{k}\right) \\
& \approx \sum_{k \in \mathbf{Z}}\left(U^{-1}\left(x_{k}\right) \int_{x_{k-1}}^{x_{k}}|f(y)| d y+\int_{x_{k}}^{x_{k+1}}|f(y)| U^{-1}(y) d y\right)^{q} \varphi\left(x_{k}\right) \\
& \approx \sum_{k \in \mathbf{Z}}\left(\int_{x_{k}}^{x_{k+1}}|f(y)| U^{-1}(y) \varphi(y)^{1 / q} d y\right)^{q} .
\end{aligned}
$$


Lemma 2.7. ([9, Lemma 3.7]) Let $q \in(0, \infty)$, let $U$ be an admissible function and let $\nu$ be a non-degenerate positive Borel measure. Moreover, let $\varphi$ be the fundamental function of $\nu$ with respect to $U^{q}$ and let $f$ be a measurable function on $[0, \infty)$. If $\left\{x_{k}\right\}$ is a discretising sequence for $\varphi$ with respect to $U^{q}$, then

$$
\begin{aligned}
\int_{[0, \infty)}\left(\sup _{y \in(0, \infty)} \frac{|f(y)|}{U(x)+U(y)}\right)^{q} d \nu(x) \\
\quad \approx \sum_{k \in \mathbf{Z}}\left(\sup _{y \in(0, \infty)} \frac{|f(y)|}{U\left(x_{k}\right)+U(y)}\right)^{q} \varphi\left(x_{k}\right) \\
\quad \approx \sum_{k \in \mathbf{Z}}\left(U^{-1}\left(x_{k}\right) \sup _{x_{k-1} \leqslant y<x_{k}}|f(y)|+\sup _{x_{k} \leqslant y<x_{k+1}}|f(y)| U(y)^{-1}\right)^{q} \varphi\left(x_{k}\right) \\
\quad \approx \sum_{k \in \mathbf{Z}} \sup _{x_{k} \leqslant y<x_{k+1}}|f(y)|^{q} U(y)^{-q} \varphi(y) .
\end{aligned}
$$

LEMMA 2.8. ([9, Lemma 3.8]) Let $v$ be a weight function, let $U$ be an admissible function and $\varphi$ be defined by (2.1). Moreover, let $\left\{x_{k}\right\}$ be a discretising sequence for $\varphi$ with respect to $U$. Then

$$
\begin{aligned}
\underset{x \in(0, \infty)}{\operatorname{esssup}}( & \left.\int_{0}^{\infty} \frac{|f(t)| d t}{U(t)+U(x)}\right) v(x) \\
& \approx \sup _{k \in \mathbf{Z}}\left(\int_{0}^{\infty} \frac{|f(t)| d t}{U(t)+U\left(x_{k}\right)}\right) \varphi\left(x_{k}\right) \\
& \approx \sup _{k \in \mathbf{Z}}\left(U^{-1}\left(x_{k}\right) \int_{x_{k-1}}^{x_{k}}|f(y)| d y+\int_{x_{k}}^{x_{k+1}}|f(y)| U^{-1}(y) d y\right) \varphi\left(x_{k}\right) \\
& \approx \sup _{k \in \mathbf{Z}}\left(\int_{x_{k}}^{x_{k+1}}|f(y)| U^{-1}(y) \varphi(y) d y\right) .
\end{aligned}
$$

Lemma 2.9. ([9, Lemma 3.9]) Let $U$ be an admissible function and let $\varphi \in \Omega_{U}$. Let $\left\{x_{k}\right\}$ be a discretising sequence for $\varphi$ with respect to $U$. Then

$$
\begin{aligned}
\sup _{x \in(0, \infty)} & \varphi(x) \sup _{0<y<\infty} \frac{|f(y)|}{U(x)+U(y)} \\
& \approx \sup _{k \in \mathbf{Z}} \varphi\left(x_{k}\right) \sup _{0<y<\infty} \frac{|f(y)|}{U\left(x_{k}\right)+U(y)} \\
& \approx \sup _{k \in \mathbf{Z}} \varphi\left(x_{k}\right) U\left(x_{k}\right)^{-1} \sup _{x_{k-1}<y<x_{k}}|f(y)|+\sup _{k \in \mathbf{Z}} \varphi\left(x_{k}\right) \sup _{x_{k}<y<x_{k+1}}|f(y)| U(y)^{-1} \\
& \approx \sup _{k \in \mathbf{Z}} \sup _{x_{k} \leqslant y<x_{k+1}}|f(y)| U(y)^{-1} \varphi(y) .
\end{aligned}
$$

Proposition 2.1. ([9, Proposition 4.1]) Let $\left\{w_{k}\right\}$ and $\left\{v_{k}\right\}, k \in \mathbb{Z}$, be two sequences of positive real numbers. Let $p, q \in(0, \infty)$ and assume that the inequality

$$
\left(\sum_{k \in \mathbf{Z}} a_{k}^{q} v_{k}\right)^{1 / q} \leqslant C\left(\sum_{k \in \mathbf{Z}} a_{k}^{p} w_{k}\right)^{1 / p}
$$

is satisfied for every sequence $\left\{a_{k}\right\}$ of positive real numbers. 
(i) If $p \leqslant q$, then

$$
\sup _{k \in \mathbf{Z}} w_{k}^{-(q / p)} v_{k}<\infty .
$$

(ii) If $p>q$ and $r=(p q) /(p-q)$, then

$$
\left(\sum_{k \in \mathbf{Z}} w_{k}^{-(r / p)} v_{k}^{r / q}\right)^{1 / r}<\infty
$$

LEMMA 2.10. ([14] and [19]) We have the following Hardy type inequalities:

(a) For the case $0<p \leqslant 1$,

$$
\left(\int_{x_{k-1}}^{x_{k}}\left(\int_{s}^{x_{k}} h(z) d z\right)^{1 / p} u(s) d s\right)^{p} \leqslant C\left(\int_{x_{k-1}}^{x_{k}} h(s) V(s) d s\right) ;
$$

the best constant $C \approx \sup _{x_{k-1}<t<x_{k}}\left(\int_{x_{k-1}}^{t} u(s) d s\right)^{p} V(t)^{-1}$ and the equivalent constant is independent of $x_{k}$.

(b) For $1<p<\infty$,

$$
\left(\int_{x_{k-1}}^{x_{k}}\left(\int_{s}^{x_{k}} h(z) d z\right)^{1 / p} u(s) d s\right)^{p} \leqslant C\left(\int_{x_{k-1}}^{x_{k}} h(s) V(s) d s\right)
$$

the best constant $C \approx\left(\int_{x_{k-1}}^{x_{k}}\left(\int_{x_{k-1}}^{t} u(s) d s\right)^{p^{\prime} / p} V(t)^{-p^{\prime} / p} u(t) d t\right)^{p / p^{\prime}}$ and the equivalent constant is independent of $x_{k}$.

We shall also use the following trivial inequality

$$
\int_{x_{k}}^{x_{k+1}} h(z) d z \leqslant C V\left(x_{k}\right)^{-1} \int_{x_{k}}^{x_{k+1}} h(s) V(s) d s
$$

\section{The Main Result}

Our main result reads:

THEOREM 3.1. Let $p, q \in(0, \infty)$ and let $u, v, w$ be weights. Assume that $u$ is such that $U^{q}$ is admissible and the measure $w(t) d t$ is non-degenerate with respect to $U^{q}$. Then the inequality (1.1) holds for every $f \in \mathcal{M}(\mathcal{R}, \mu)$ if and only if

(i) $0<p \leqslant 1, p \leqslant q<\infty$

$$
A_{1}:=\sup _{x>0}\left(\int_{0}^{\infty}\left(\frac{U(x)}{U(t)+U(x)}\right)^{q} w(t) d t\right)^{1 / q} V^{-1 / p}(x)<\infty .
$$

Moreover, the best constant $C$ in (1.1) satisfies $C \approx A_{1}$. 
(ii) $0<q<p \leqslant 1$

$$
\begin{aligned}
A_{2}:=\left(\int_{0}^{\infty}\left(\int_{0}^{\infty}\left(\frac{U(x)}{U(t)+U(x)}\right)^{q} w(t) d t\right)^{(r-q) / q} w(x) U(x)^{-r}\right. & \\
& \left.\times\left(\sup _{0<t<x} U(t) V(t)^{-(1 / p)}\right)^{r} d x\right)^{1 / r}<\infty
\end{aligned}
$$

Moreover, the best constant $C$ in (1.1) satisfies $C \approx A_{2}$.

(iii) $1<p \leqslant q<\infty$,

$$
\begin{aligned}
& A_{3}:=\sup _{x>0}\left(\int_{0}^{\infty}\left(\frac{U(x)}{U(t)+U(x)}\right)^{q} w(t) d t\right)^{1 / q} \times \\
&\left(\int_{0}^{\infty}\left(\frac{U(t)}{U(t)+U(x)}\right)^{p^{\prime}} \frac{v(t)}{V^{p^{\prime}}(t)} d t\right)^{1 / p^{\prime}}<\infty .
\end{aligned}
$$

Moreover, the best constant $C$ in (1.1) satisfies $C \approx A_{3}$.

(iv) $1<p<\infty, 0<q<p, r=(p q) /(p-q)$

$$
\begin{aligned}
& A_{4}:=\left(\int_{0}^{\infty}\left(\int_{0}^{\infty}\left(\frac{U(x)}{U(t)+U(x)}\right)^{q} w(t) d t\right)^{(r-q) / q} w(x)\right. \\
&\left.\times\left(\int_{0}^{\infty}\left(\frac{U(t)}{U(t)+U(x)}\right)^{p^{\prime}} \frac{v(t) d t}{V^{p^{\prime}}(t)}\right)^{r / p^{\prime}} d x\right)^{1 / r}<\infty
\end{aligned}
$$

Moreover, the best constant $C$ in (1.1) satisfies $C \approx A_{4}$.

(v) $0<p \leqslant 1, q=\infty$

$$
A_{5}:=\sup _{x>0}\left(\operatorname{esssup}_{t \in(0, \infty)}\left(\frac{w(t) U(x)}{U(t)+U(x)}\right)\right) V^{-1 / p}(x)<\infty .
$$

Moreover, the best constant $C$ in (1.1) satisfies $C \approx A_{5}$.

(vi) $1<p<\infty, q=\infty$

$$
A_{6}:=\sup _{x>0}\left(\operatorname{esssup}_{t \in(0, \infty)} \frac{w(t) U(x)}{U(t)+U(x)}\right)\left(\int_{0}^{\infty}\left(\frac{U(t)}{U(t)+U(x)}\right)^{p^{\prime}} \frac{v(t)}{V^{p^{\prime}}(t)} d t\right)^{1 / p^{\prime}}<\infty .
$$

Moreover, the best constant $C$ in (1.1) satisfies $C \approx A_{6}$.

(vii) $p=q=\infty$

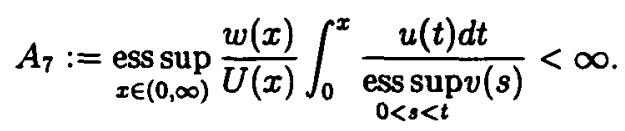

Moreover, the best constant $C$ in (1.1) satisfies $C \approx A_{7}$.

(viii) $p=\infty, 0<q<\infty$

$$
A_{8}:=\left(\int_{0}^{\infty}\left(\frac{1}{U(x)} \int_{0}^{x} \frac{u(t) d t}{\underset{0<s<t}{\operatorname{esssup} v(s)}}\right)^{q} w(x) d x\right)^{1 / q}<\infty .
$$

Moreover, the best constant $C$ in (1.1) satisfies $C \approx A_{8}$. 
Our next goal is to formulate Theorem 3.1 in a more compact and nice form. We need the following definition of the associate space (also called the Köthe dual) $\Lambda^{p}(v)_{u}^{\prime}$ of $\Lambda^{p}(v)$.

Definition 3.1: Let $0<p \leqslant \infty$ and let $u, v$ be weight functions. The $u$-associate or Köthe dual space $\Lambda^{p}(v)_{u}^{\prime}$ of $\Lambda^{p}(v)$ is defined by

$$
\|f\|_{\Lambda^{p}(v)_{u}^{\prime}}=: \sup _{g \in \Lambda^{p}(v)} \frac{\int_{0}^{\infty} f^{*}(t) g^{*}(t) u(t) d t}{\|g\|_{\Lambda^{p}(v)}}<\infty .
$$

In the classical situation (that is when $u=1$ ) the associate norm $\Lambda^{p}(v)^{\prime}$ was characterised by Sawyer [15] for $1<p<\infty$ and by Stepanov [20] for $0<p \leqslant 1$. In the case $p=\infty$ it was considered in [7] (see also [8]). In fact we can describe $\Lambda^{p}(v)^{\prime}$ in the following way:

$$
\Lambda^{p}(v)^{\prime}= \begin{cases}\Gamma^{\infty}\left(\frac{t}{V(t)^{1 / p}}\right), & 0<p \leqslant 1 \\ \Gamma^{p^{\prime}}\left(\frac{t^{p^{\prime}} v(t)}{V}(t)^{p^{\prime}}\right), & 1<p<\infty \\ \Lambda^{1}\left(\frac{1}{\operatorname{ess~sup}_{0<s<t} v(s)}\right), & p=\infty .\end{cases}
$$

REMARK 3.1. By letting $t \mapsto U^{-1}(t)$ (where $U^{-1}$ is the inverse function of $U$ ) in (3.1) and after using (3.2) we can obtain the following characterisation of $\Lambda^{p}(v)_{u}^{\prime}$.

$$
\Lambda^{p}(v)_{u}^{\prime}= \begin{cases}\Gamma_{u}^{\infty}\left(\frac{U(t)}{V(t)^{1 / p}}\right), & 0<p \leqslant 1 \\ \Gamma_{u}^{p^{\prime}}\left(\frac{U(t)^{p^{\prime}} v(t)}{V(t)^{p^{\prime}}}\right), & 1<p<\infty \\ \Lambda^{1}\left(\frac{u(t)}{\operatorname{ess~sup}_{0<s<t}(s)}\right), & p=\infty .\end{cases}
$$

Using $\Gamma_{u}^{q}(w)$ and $\Lambda^{p}(v)_{u}^{\prime}$ we can now formulate Theorem 3.1 in the following compact form:

THEOREM 3.2. Let $p, q \in(0, \infty]$ and let $u, v, w$ be weights. When $q \in(0, \infty)$ we assume that $u$ is such that $U^{q}$ is admissible and the measure $w(t) d t$ is non-degenerate with respect to $U^{q}$. Then the inequality (1.1) holds for every $f \in \mathcal{M}(\mathcal{R}, \mu)$ if and only if

(i) $0<p \leqslant q \leqslant \infty$

$$
\sup _{t}\|\chi(0, t)\|_{\Gamma_{u(w)}^{q}}\left\|\chi_{(0, t)}\right\|_{\Lambda^{p}(v)_{u}^{\prime}} U(t)^{-1}<\infty
$$

(ii) $0<q<p \leqslant \infty, r=(p q) /(p-q)$

$$
\left(\int_{0}^{\infty}\left\|\chi_{(0, t)}\right\|_{\Gamma_{u}^{q}(w)}^{r-q} w(t) U(t)^{-r}\left\|\chi_{(0, t)}\right\|_{\Lambda^{p}(v)_{u}^{\prime}}^{r} d t\right)^{1 / r}<\infty .
$$


REMARK 3.2. Theorem 3.1 and Theorem 3.2 are in fact equivalent, the only difference is that Theorem 3.2 is stated in a more compact form. All the eight conditions $A_{1}-A_{8}$ in Theorem 3.1 can be obtained from the two conditions in Theorem 3.2.

\section{PROOFS}

Proof of TheOREM 3.1. We start with the upper bounds (sufficiency).

Let $0<p<\infty$. First we use the fact that $f$ is non-increasing if and only if $f^{p}$ is non-increasing and a standard argument shows that it is enough to prove (1.1) for $f$ satisfying

$$
f^{*}(t)^{p}=\int_{t}^{\infty} h(s) d s
$$

where $h$ is some positive measurable function on $(0, \infty)$. That is, when $0<q<\infty$ we only have to prove that

$$
\begin{aligned}
\left(\int_{0}^{\infty}\left(\frac{1}{U(t)} \int_{0}^{t}\left(\int_{s}^{\infty} h(z) d z\right)^{1 / p} u(s) d s\right)^{q} w(t) d t\right)^{1 / q} & \\
& \leqslant C\left(\int_{0}^{\infty}\left(\int_{t}^{\infty} h(s) d s\right) v(t) d t\right)^{1 / p}
\end{aligned}
$$

holds for every non-negative function $h$, and for $q=\infty$, by the Lemma 2.2 we have to prove that the inequality

$$
\sup _{t \in(0, \infty)} \frac{\varphi(t)}{U(t)} \int_{0}^{t}\left(\int_{s}^{\infty} h(z) d z\right)^{1 / p} u(s) d s \leqslant C\left(\int_{0}^{\infty}\left(\int_{t}^{\infty} h(s) d s\right) v(t) d t\right)^{1 / p}
$$

holds for every non-negative function $h$, where the function $\varphi$ is defined by (2.1).

According to Fubini's theorem the inequalities (4.1) and (4.2) are equivalent to

$$
\left(\int_{0}^{\infty}\left(\frac{1}{U(t)} \int_{0}^{t}\left(\int_{s}^{\infty} h(z) d z\right)^{1 / p} u(s) d s\right)^{q} w(t) d t\right)^{1 / q} \leqslant C\left(\int_{0}^{\infty} h(t) V(t) d t\right)^{1 / p}
$$

and

$$
\sup _{t \in(0, \infty)} \frac{\varphi(t)}{U(t)} \int_{0}^{t}\left(\int_{s}^{\infty} h(z) d z\right)^{1 / p} u(s) d s \leqslant C\left(\int_{0}^{\infty} h(t) V(t) d t\right)^{1 / p}
$$

respectively.

Assume first that $0<q<\infty$. Define

$$
\varphi(x)=U^{q}(x) \int_{0}^{\infty} \frac{w(s) d s}{(U(s)+U(x))^{q}} .
$$


Then $\varphi \in \Omega_{U^{q}}$ and therefore there exists a discretising sequence for $\varphi$ with respect to $U^{q}$. Let $\left\{x_{k}\right\}$ be one such sequence. Then $\varphi\left(x_{k}\right) \Uparrow$ and $\left(\varphi\left(x_{k}\right)\right) /\left(U^{q}\left(x_{k}\right)\right) \Downarrow$. Furthermore, there is a decomposition $\mathbb{Z}=\mathbb{Z}_{1} \cup \mathbb{Z}_{2}, \mathbb{Z}_{1} \cap \mathbb{Z}_{2}=\emptyset$ such that for every $k \in \mathbb{Z}_{1}$ and $t \in\left[x_{k}, x_{k+1}\right], \varphi\left(x_{k}\right) \approx \varphi(t)$ and for every $k \in \mathbb{Z}_{2}$ and

$$
t \in\left[x_{k}, x_{k+1}\right], \frac{\varphi\left(x_{k}\right)}{U^{q}\left(x_{k}\right)} \approx \frac{\varphi(t)}{U^{q}(t)} .
$$

For the left-hand side of (4.1), using Lemma 2.3 with $d v(t)=w(t) d t$ and $f(t)=\int_{0}^{t}\left(\int_{s}^{\infty} h(z) d z\right)^{1 / p} u(s) d s$ we get that

$$
\begin{aligned}
\left(\int_{0}^{\infty}\left(\frac{1}{U(t)} \int_{0}^{t}\left(\int_{s}^{\infty} h(z) d z\right)^{1 / p} u(s) d s\right)^{q} w(t) d t\right)^{1 / q} & \\
& \approx\left(\sum_{k \in \mathbf{Z}}\left(\int_{0}^{x_{k}}\left(\int_{s}^{\infty} h(z) d z\right)^{1 / p} u(s) d s\right)^{q} \frac{\varphi\left(x_{k}\right)}{U\left(x_{k}\right)^{q}}\right)^{1 / q}
\end{aligned}
$$

[using Lemma 2.5]

$$
\begin{aligned}
& \approx\left(\sum_{k \in \mathbf{Z}}\left(\int_{x_{k-1}}^{x_{k}}\left(\int_{s}^{\infty} h(z) d z\right)^{1 / p} u(s) d s\right)^{q} \frac{\varphi\left(x_{k}\right)}{U\left(x_{k}\right)^{q}}\right)^{1 / q} \\
& =\left(\sum_{k \in \mathbf{Z}}\left(\int_{x_{k-1}}^{x_{k}}\left(\int_{s}^{x_{k}} h(z) d z+\int_{x_{k}}^{\infty} h(z) d z\right)^{1 / p} u(s) d s\right)^{q} \frac{\varphi\left(x_{k}\right)}{U\left(x_{k}\right)^{q}}\right)^{1 / q} \\
& \approx\left(\sum_{k \in \mathbf{Z}}\left(\int_{x_{k-1}}^{x_{k}}\left(\int_{s}^{x_{k}} h(z) d z\right)^{1 / p} u(s) d s\right)^{q} \frac{\varphi\left(x_{k}\right)}{U\left(x_{k}\right)^{q}}\right)^{1 / q} \\
& +\left(\sum_{k \in \mathbf{Z}}\left(\int_{x_{k-1}}^{x_{k}}\left(\int_{x_{k}}^{\infty} h(z) d z\right)^{1 / p} u(s) d s\right)^{q} \frac{\varphi\left(x_{k}\right)}{U\left(x_{k}\right)^{q}}\right)^{1 / q} \\
& \approx\left(\sum_{k \in \mathbf{Z}}\left(\int_{x_{k-1}}^{x_{k}}\left(\int_{s}^{x_{k}} h(z) d z\right)^{1 / p} u(s) d s\right)^{q} \frac{\varphi\left(x_{k}\right)}{U\left(x_{k}\right)^{q}}\right)^{1 / q} \\
& \quad+\left(\sum_{k \in \mathbf{Z}}\left(\int_{x_{k}}^{\infty} h(z) d z\right)^{q / p}\left(\int_{x_{k-1}}^{x_{k}} u(s) d s\right)^{q} \frac{\varphi\left(x_{k}\right)}{U\left(x_{k}\right)^{q}}\right)^{1 / q}
\end{aligned}
$$

[using the fact that $\int_{x_{k-1}}^{x_{k}} u(s) d s=U\left(x_{k}\right)-U\left(x_{k-1}\right) \approx U\left(x_{k}\right)$ ]

$$
\begin{aligned}
\approx\left(\sum_{k \in \mathbf{Z}}\left(\int_{x_{k-1}}^{x_{k}}\left(\int_{s}^{x_{k}} h(z) d z\right)^{1 / p} u(s) d s\right)^{q} \frac{\varphi\left(x_{k}\right)}{U\left(x_{k}\right)^{q}}\right)^{1 / q} & \\
& +\left(\sum_{k \in \mathbf{Z}}\left(\int_{x_{k}}^{\infty} h(z) d z\right)^{q / p} \varphi\left(x_{k}\right)\right)^{1 / q}
\end{aligned}
$$


[using Lemma 2.5 on the second term]

$$
\begin{aligned}
\approx\left(\sum_{k \in \mathbf{Z}}\left(\int_{x_{k-1}}^{x_{k}}\left(\int_{s}^{x_{k}} h(z) d z\right)^{1 / p} u(s) d s\right)^{q} \frac{\varphi\left(x_{k}\right)}{U\left(x_{k}\right)^{q}}\right)^{1 / q} \\
+\left(\sum_{k \in Z}\left(\int_{x_{k}}^{x_{k+1}} h(z) d z\right)^{q / p} \varphi\left(x_{k}\right)\right)^{1 / q}=I+I I .
\end{aligned}
$$

Now we shall distinguish several cases. Starting with the case $0<p \leqslant 1$. Using (2.2) for $I$ and (2.4) for $I I$ we find that

$$
\begin{aligned}
I= & \left(\sum_{k \in \mathbf{Z}}\left(\int_{x_{k-1}}^{x_{k}}\left(\int_{s}^{x_{k}} h(z) d z\right)^{1 / p} u(s) d s\right)^{q} \frac{\varphi\left(x_{k}\right)}{U\left(x_{k}\right)^{q}}\right)^{1 / q} \\
\leqslant & \left(\sum_{k \in \mathbf{Z}} \frac{\varphi\left(x_{k}\right)}{U\left(x_{k}\right)^{q}}\left(\sup _{x_{k-1}<t<x_{k}}\left(\int_{x_{k-1}}^{t} u(s) d s\right) V(t)^{-1 / p}\right)^{q}\right. \\
& \left.\times\left(\int_{x_{k-1}}^{x_{k}} h(s) V(s) d s\right)^{q / p}\right)^{1 / q} \\
\leqslant & \left(\sum_{k \in \mathbf{Z}} \frac{\varphi\left(x_{k}\right)}{U\left(x_{k}\right)^{q}}\left(\sup _{x_{k-1}<t<x_{k}} U(t)^{q} V(t)^{-(q / p)}\right)\left(\int_{x_{k-1}}^{x_{k}} h(s) V(s) d s\right)^{q / p}\right)^{1 / q}
\end{aligned}
$$

and

$$
\begin{aligned}
I I & =:\left(\sum_{k \in \mathbf{Z}}\left(\int_{x_{k}}^{x_{k+1}} h(z) d z\right)^{q / p} \varphi\left(x_{k}\right)\right)^{1 / q} \\
& \leqslant\left(\sum_{k \in \mathbf{Z}} \varphi\left(x_{k}\right) V\left(x_{k}\right)^{-(q / p)}\left(\int_{x_{k}}^{x_{k+1}} h(s) V(s) d s\right)^{q / p}\right)^{1 / q} .
\end{aligned}
$$

(i) In the case $0<p \leqslant 1, p \leqslant q<\infty$ (that is $q / p \geqslant 1$ ) we have according to (4.6) that

$$
I \leqslant \sup _{k} \frac{\varphi\left(x_{k}\right)^{1 / q}}{U\left(x_{k}\right)} \sup _{x_{k-1}<t<x_{k}} U(t) V(t)^{-(1 / p)}\left(\int_{0}^{\infty} h(s) V(s) d s\right)^{1 / p},
$$

and, similarly, if $q / p \geqslant 1$ we have according to (4.7) that

$$
I \dot{I} \leqslant \sup _{k} \varphi\left(x_{k}\right)^{1 / q} V\left(x_{k}\right)^{-(1 / p)}\left(\int_{0}^{\infty} h(s) V(s) d s\right)^{1 / p}
$$

and, finally, according to (4.4) and Lemma 2.9, we get that

$$
\begin{aligned}
I+I I & \leqslant \sup _{k} \frac{\varphi\left(x_{k}\right)^{1 / q}}{U\left(x_{k}\right)}\left(\sup _{x_{k-1}<t<x_{k}} U(t) V(t)^{-1 / p}\right)\left(\int_{0}^{\infty} h(s) V(s) d s\right)^{1 / p} \\
& =A_{1}\left(\int_{0}^{\infty} h(s) V(s) d s\right)^{1 / p} .
\end{aligned}
$$


(ii) For the case $0<q<p \leqslant 1, r=(p q) /(p-q)$, by applying Hölder's inequality for sums to the right-hand side of (4.6) and the right hand side of (4.7) with exponents $p / q$ and $r / q$, we find that

$$
\begin{aligned}
I & \lesssim\left(\sum_{k \in \mathbf{Z}} \frac{\varphi\left(x_{k}\right)^{r / q}}{U\left(x_{k}\right)^{r}}\left(\sup _{x_{k-1}<t<x_{k}} U(t) V(t)^{-(1 / p)}\right)^{r}\right)^{1 / r}\left(\int_{0}^{\infty} h(s) V(s) d s\right)^{1 / p} \\
I I & \lesssim\left(\sum_{k \in \mathbf{Z}} \varphi\left(x_{k}\right)^{r / q} V\left(x_{k}\right)^{-(r / p)}\right)^{1 / r}\left(\int_{0}^{\infty} h(s) V(s) d s\right)^{1 / p} .
\end{aligned}
$$

Therefore, we get that

$$
I+I I \lesssim\left(\sum_{k \in \mathbf{Z}} \frac{\varphi\left(x_{k}\right)^{r / q}}{U\left(x_{k}\right)^{r}}\left(\sup _{x_{k-1}<t<x_{k}} U(t) V(t)^{-(1 / p)}\right)^{r}\right)^{1 / r} \times\left(\int_{0}^{\infty} h(s) V(s) d s\right)^{q / p}
$$

By using the Lemma 2.7 we obtain from (4.8) that

$$
I+I I \lesssim A_{2}\left(\int_{0}^{\infty} h(s) V(s) d s\right)^{1 / p} .
$$

Now let us assume that $1<p<\infty$. Using (2.3) for $I$ and (2.4) for $I I$, we get

$$
\begin{gathered}
I \lesssim\left(\sum_{k \in \mathbf{Z}} \frac{\varphi\left(x_{k}\right)}{U\left(x_{k}\right)^{q}}\left(\int_{x_{k-1}}^{x_{k}} U(t)^{p^{\prime} / p} V(t)^{-p^{\prime} / p} u(t) d t\right)^{q / p^{\prime}}\right. \\
\left.\times\left(\int_{x_{k-1}}^{x_{k}} h(s) V(s) d s\right)^{q / p}\right)^{1 / q} \\
I I \lesssim\left(\sum_{k \in \mathbf{Z}} \varphi\left(x_{k}\right) V\left(x_{k}\right)^{-q / p}\left(\int_{x_{k}}^{x_{k+1}} h(s) V(s) d s\right)^{q / p}\right)^{1 / q} .
\end{gathered}
$$

(iii) Suppose that $1<p \leqslant q<\infty$. Then, according to (4.9) and (4.10), we obtain that

$$
\begin{aligned}
& I \lesssim \sup _{k>0} \frac{\varphi\left(x_{k}\right)^{1 / q}}{U\left(x_{k}\right)}\left(\int_{x_{k-1}}^{x_{k}} U(t)^{p^{\prime} / p} V(t)^{-p^{\prime} / p} u(t) d t\right)^{1 / p^{\prime}}\left(\int_{0}^{\infty} h(s) V(s) d s\right)^{1 / p}, \\
& I I \lesssim \sup _{k} \varphi\left(x_{k}\right)^{1 / q} V\left(x_{k}\right)^{-1 / p}\left(\int_{0}^{\infty} h(s) V(s) d s\right)^{1 / p} .
\end{aligned}
$$

Hence, using integration by part and Lemma 2.8 , we get that

$$
\begin{gathered}
I+I I \lesssim \sup _{k>0} \frac{\varphi\left(x_{k}\right)^{1 / q}}{U\left(x_{k}\right)}\left(\int_{x_{k-1}}^{x_{k}} V(t)^{-p^{\prime} / p} U(t)^{p^{\prime} / p} u(t) d t+U\left(x_{k}\right)^{p^{\prime}} V\left(x_{k}\right)^{-\left(p^{\prime} / p\right)}\right)^{1 / p^{\prime}} \\
\times\left(\int_{0}^{\infty} h(s) V(s) d s\right)^{1 / p}
\end{gathered}
$$




$$
\begin{aligned}
& \lesssim \sup _{k>0} \frac{\varphi\left(x_{k}\right)^{1 / q}}{U\left(x_{k}\right)}\left(\int_{x_{k-1}}^{x_{k}} V(t)^{-p^{\prime}} U(t)^{p^{\prime}} v(t) d t+V\left(x_{k}\right)^{-\left(p^{\prime} / p\right)} U\left(x_{k}\right)^{p^{\prime}}\right)^{1 / p^{\prime}} \\
& \quad \times\left(\int_{0}^{\infty} h(s) V(s) d s\right)^{1 / p} \\
& \lesssim \sup _{k>0} \varphi\left(x_{k}\right)^{1 / q}\left(\int_{0}^{\infty} \frac{U(t)^{p^{\prime}} V(t)^{-p^{\prime}} v(t) d t}{\left(U(t)+U\left(x_{k}\right)\right)^{p^{\prime}}}\right)^{1 / p^{\prime}}\left(\int_{0}^{\infty} h(s) V(s) d s\right)^{1 / p} \\
& =A_{3}\left(\int_{0}^{\infty} h(s) V(s) d s\right)^{1 / p} .
\end{aligned}
$$

(iv) Next we consider the case $1<p<\infty, 0<q<p, r=(p q) /(p-q)$. By using the Hölder's inequality for sums to the right-hand side of (4.9) and the right hand side of (4.10) with exponents $p / q$ and $r / q$, we find that

$$
I \lesssim\left(\sum_{k \in \mathbf{Z}} \frac{\varphi^{r / q}\left(x_{k}\right)}{U\left(x_{k}\right)^{r}}\left(\int_{x_{k-1}}^{x_{k}} U(t)^{p^{\prime} / p} V\left(x_{k}\right)^{-\left(p^{\prime} / p\right)} u(t) d t\right)^{r / p^{\prime}}\right)^{1 / r}\left(\int_{0}^{\infty} h(s) V(s) d s\right)^{1 / p}
$$

and

$$
I I \lesssim\left(\sum_{k \in \mathbf{Z}} \varphi^{r / q}\left(x_{k}\right) V\left(x_{k}\right)^{-(r / p)}\right)^{1 / r}\left(\int_{0}^{\infty} h(s) V(s) d s\right)^{1 / p}
$$

Therefore, using integration by part and Lemma 2.6 , we see that

$$
\begin{aligned}
I+I I & \lesssim\left(\sum_{k \in \mathbf{Z}} \frac{\varphi^{r / q}\left(x_{k}\right)}{U\left(x_{k}\right)^{r}}\left(\int_{x_{k-1}}^{x_{k}} V(t)^{-\left(p^{\prime} / p\right)} U(t)^{p^{\prime} / p} u(t) d t+V\left(x_{k}\right)^{-\left(p^{\prime} / p\right)} U\left(x_{k}\right)^{p^{\prime}}\right)^{r / p^{\prime}}\right)^{1 / r} \\
& \lesssim\left(\int_{0 \in \mathbf{Z}}^{\infty} \frac{\varphi^{r / q}\left(x_{k}\right)}{U\left(x_{k}\right)^{r}}\left(\int_{x_{k-1}}^{x_{k}} V(t)^{-p^{\prime}} U(t)^{p^{\prime}} v(t) d t+V\left(x_{k}\right)^{-\left(p^{\prime} / p\right)} U\left(x_{k}\right)^{p^{\prime}}\right)^{r / p}\right)^{1 / r} \\
& \lesssim\left(\sum_{k \in \mathbf{Z}} \varphi^{r / q}\left(x_{k}\right)\left(\int_{0}^{\infty} \frac{U(t)^{p^{\prime}} V(t)^{-p^{\prime}} v(t) d t}{\left(U(t)+U\left(x_{k}\right)\right)^{p^{\prime}}}\right)^{r / p^{\prime}}\right)^{1 / r}\left(\int_{0}^{\infty} h(s) V(s) d s\right)^{1 / p} \\
& \leqslant A_{4}\left(\int_{0}^{\infty} h(s) V(s) d s\right)^{1 / p} \cdot
\end{aligned}
$$

Let now $q=\infty$. In the same way, by using Lemma 2.2, Lemma 2.4 and Lemma 2.5 we obtain for the left-hand side of (4.3) that

$$
\sup _{t \in(0, \infty)} \frac{\varphi(t)}{U(t)} \int_{0}^{t}\left(\int_{s}^{\infty} h(z) d z\right)^{1 / p} u(s) d s
$$




$$
\begin{aligned}
& \approx \sup _{k \in \mathbf{Z}} \frac{\varphi\left(x_{k}\right)}{U\left(x_{k}\right)} \int_{0}^{x_{k}}\left(\int_{s}^{\infty} h(z) d z\right)^{1 p} u(s) d s \\
& \approx \sup _{k \in \mathbf{Z}} \frac{\varphi\left(x_{k}\right)}{U\left(x_{k}\right)} \int_{x_{k-1}}^{x_{k}}\left(\int_{s}^{x_{k}} h(z) d z\right)^{1 / p} u(s) d s \\
& +\sup _{k \in \mathbf{Z}} \varphi\left(x_{k}\right)\left(\int_{x_{k}}^{x_{k+1}} h(z) d z\right)^{1 p}:=I I I+I V,
\end{aligned}
$$

where $\varphi$ is defined by (2.1).

(v) Let $0<p \leqslant 1, q=\infty$. By using the inequality (2.2) for $I I I$ and (2.4) for $I V$, we arrive at

$$
\begin{aligned}
& I I I \lesssim \sup _{k} \frac{\varphi\left(x_{k}\right)}{U\left(x_{k}\right)}\left(\sup _{x_{k-1}<t<x_{k}} U(t) V(t)^{-(1 / p)}\right)\left(\int_{x_{k}}^{x_{k+1}} h(s) V(s) d s\right)^{1 / p}, \\
& I V \lesssim \sup _{k} \varphi\left(x_{k}\right) V\left(x_{k}\right)^{-q / p}\left(\int_{x_{k}}^{x_{k+1}} h(s) V(s) d s\right)^{1 / p}
\end{aligned}
$$

and, finally, by using Lemma 2.9 we get that

$$
\begin{aligned}
I I I+I V & \lesssim \sup _{k} \frac{\varphi\left(x_{k}\right)}{U\left(x_{k}\right)}\left(\sup _{x_{k-1}<t<x_{k}} U(t) V(t)^{-1 / p}\right)\left(\int_{0}^{\infty} h(s) V(s) d s\right)^{1 / p} \\
& \lesssim A_{5}\left(\int_{0}^{\infty} h(s) V(s) d s\right)^{1 / p} .
\end{aligned}
$$

(vi) Let now $1<p<\infty, q=\infty$. By using (2.3) for $I I I$ and (2.4) for $I V$, we obtain that

$$
I I I \lesssim\left(\sup _{k \in \mathbf{Z}} \frac{\varphi\left(x_{k}\right)}{U\left(x_{k}\right)}\left(\int_{x_{k-1}}^{x_{k}} U(t)^{p^{\prime} / p} V(t)^{-p^{\prime} / p} u(t) d t\right)^{1 / p^{\prime}} \times\left(\int_{x_{k-1}}^{x_{k}} h(s) V(s) d s\right)^{1 / p}\right)
$$

and

$$
I V \lesssim\left(\sum_{k \in \mathbf{Z}} \varphi\left(x_{k}\right) V\left(x_{k}\right)^{-1 / p}\left(\int_{x_{k}}^{x_{k+1}} h(s) V(s) d s\right)^{1 / p}\right) .
$$

Using integration by part and Lemma 2.8, we find that

$$
\begin{gathered}
I I I+I V \lesssim \sup _{k>0} \frac{\varphi\left(x_{k}\right)}{U\left(x_{k}\right)}\left(\int_{x_{k-1}}^{x_{k}} V(t)^{-\left(p^{\prime} / p\right)} U(t)^{p^{\prime} / p} u(t) d t+V\left(x_{k}\right)^{-\left(p^{\prime} / p\right)} U\left(x_{k}\right)^{p^{\prime}}\right)^{1 / p^{\prime}} \\
\times\left(\int_{0}^{\infty} h(s) V(s) d s\right)^{1 / p} \\
\lesssim \sup _{k>0} \frac{\varphi\left(x_{k}\right)}{U\left(x_{k}\right)}\left(\int_{x_{k-1}}^{x_{k}} V(t)^{-p^{\prime}} U(t)^{p^{\prime}} v(t) d t+V\left(x_{k}\right)^{-\left(p^{\prime} / p\right)} U\left(x_{k}\right)^{p^{\prime}}\right)^{1 / p^{\prime}} \\
\times\left(\int_{0}^{\infty} h(s) V(s) d s\right)^{1 / p}
\end{gathered}
$$




$$
\begin{aligned}
& \lesssim \sup _{k>0} \varphi\left(x_{k}\right)\left(\int_{0}^{\infty} \frac{U(t)^{p^{\prime}} V(t)^{-p^{\prime}} v(t) d t}{U(t)^{p^{\prime}}+U\left(x_{k}\right)^{p^{\prime}}}\right)^{1 / p^{\prime}} \times\left(\int_{0}^{\infty} h(s) V(s) d s\right)^{1 / p} \\
& =A_{6}\left(\int_{0}^{\infty} h(s) V(s) d s\right)^{1 / p} .
\end{aligned}
$$

(vii) For the case $p=q=\infty$, we use Lemma 2.1 and get that

$$
\begin{aligned}
\|f\|_{\Gamma_{u}^{\infty}(w)} & =\underset{x \in(0, \infty)}{\operatorname{ess~sup}} \frac{w(x)}{U(x)} \int_{0}^{x} f^{*}(t) u(t) d t \\
& \leqslant \underset{x \in(0, \infty)}{\operatorname{ess~supp}} \frac{w(x)}{U(x)} \int_{0}^{x} \frac{u(t) d t}{\operatorname{ess} \sup _{0<s<t} v(s)} \underset{x \in(0, \infty)}{\operatorname{ess~sup}} f^{*}(x) \underset{0<s<x}{\operatorname{ess} \sup } v(s) \\
& \leqslant A_{7}\|f\|_{\Lambda^{\infty}(v)} .
\end{aligned}
$$

(viii) Finally, for $p=\infty, 0<q<\infty$, by Lemma 2.1 we obtain that

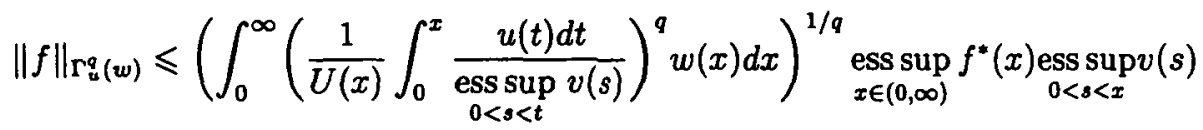

$$
\begin{aligned}
& \leqslant A_{8}\|f\|_{\Lambda^{\infty}(v)} \text {. }
\end{aligned}
$$

Next we prove the lower bounds (Necessity).

Let $0<q<\infty$ and let $\left\{x_{k}\right\}$ be a discretising sequence for $\varphi$ from (4.4). Then by (4.5) we have that

$$
\begin{aligned}
\left(\sum_{k \in \mathbf{Z}}\left(\int_{x_{k-1}}^{x_{k}}\left(\int_{s}^{x_{k}} h(z) d z\right)^{1 / p} u(s) d s\right)^{q} \frac{\varphi\left(x_{k}\right)}{U\left(x_{k}\right)^{q}}\right)^{1 / q} \\
+\left(\sum_{k \in \mathbf{Z}}\left(\int_{x_{k}}^{x_{k+1}} h(z) d z\right)^{q / p} \varphi\left(x_{k}\right)\right)^{1 q} \lesssim\left(\sum_{k \in \mathbf{Z}} \int_{x_{k-1}}^{x_{k}} h(t) V(t) d t\right)^{1 / p} .
\end{aligned}
$$

Let $0<p \leqslant 1$. For $k \in \mathbb{Z}$, let $h_{k}$ be functions that satisfy the Hardy inequality (2.2) and the Hölder inequality (2.4), that is, functions $h_{k}$ satisfying

$$
\begin{aligned}
& \operatorname{supp} h_{k} \subset\left[x_{k-1}, x_{k}\right], \quad \int_{x_{k-1}}^{x_{k}} h_{k}(s) V(s) d s=1, \\
& \left(\int_{x_{k-1}}^{x_{k}}\left(\int_{s}^{x_{k}} h_{k}(t) d t\right)^{1 / p} u(s) d s\right)^{p} \gtrsim \sup _{x_{k-1}<t<x_{k}}\left(U(t)-U\left(x_{k-1}\right)\right)^{p} V(t)^{-1} \text {, } \\
& \int_{x_{k-1}}^{x_{k}} h_{k}(s) d s \gtrsim V\left(x_{k-1}\right)^{-1} .
\end{aligned}
$$

Now we define the test function

$$
h(s)=\sum_{k \in \mathbf{Z}} a_{k} h_{k}(s)
$$


where $\left\{a_{k}\right\}$ is a sequence of positive real numbers. Then, by using this test function in (4.12), we get that

$$
\begin{aligned}
\left(\sum_{k \in \mathbf{Z}} a_{k}^{q / p} \frac{\varphi\left(x_{k}\right)}{U\left(x_{k}\right)^{q}} \sup _{x_{k-1}<t<x_{k}}\right. & \left.\left(U(t)-U\left(x_{k-1}\right)\right)^{q} V(t)^{-q / p}\right)^{1 / q} \\
& +\left(\sum_{k \in \mathbf{Z}} a_{k}^{q / p} \varphi\left(x_{k-1}\right) V\left(x_{k-1}\right)^{-(q / p)}\right)^{1 / q} \lesssim\left(\sum_{k \in \mathbf{Z}} a_{k}\right)^{1 / p} .
\end{aligned}
$$

Now, by using Proposition 2.1 for the case $0<p \leqslant 1, p \leqslant q$, we find that

$$
\sup _{k \in \mathbf{Z}} \varphi\left(x_{k}\right)^{1 / q} V\left(x_{k}\right)^{-1 / p}+\sup _{k \in \mathbf{Z}}\left(\sup _{x_{k-1}<t<x_{k}}\left(U(t)-U\left(x_{k-1}\right)\right) V(t)^{-1 / p}\right) \frac{\varphi\left(x_{k}\right)^{1 / q}}{U\left(x_{k}\right)}<\infty .
$$

Moreover, according to Lemma 2.9 , we see that

$$
\begin{aligned}
A_{1} & \lesssim \sup _{k \in \mathbf{Z}} \sup _{x_{k-1}<t<x_{k}} V(t)^{-1 / p} \varphi(t)^{1 / q} \\
& \lesssim \sup _{k \in \mathbf{Z}} \varphi\left(x_{k}\right)^{1 / q} V\left(x_{k}\right)^{-1 / p}+\sup _{k \in \mathbf{Z}}\left(\sup _{x_{k-1}<t<x_{k}} U(t) V(t)^{-1 / p}\right) \frac{\varphi\left(x_{k}\right)^{1 / q}}{U\left(x_{k}\right)} \\
& \lesssim \sup _{k \in \mathbf{Z}} \varphi\left(x_{k}\right)^{1 / q} V\left(x_{k}\right)^{-1 / p} \\
& \quad+\sup _{k \in \mathbf{Z}}\left(\sup _{x_{k-1}<t<x_{k}}\left(U(t)-U\left(x_{k-1}\right)\right) V(t)^{-1 / p}\right) \frac{\varphi\left(x_{k}\right)^{1 / q}}{U\left(x_{k}\right)}<\infty .
\end{aligned}
$$

Let $0<q<p \leqslant 1$. We obtain from (4.14) and Proposition 2.1 that

$$
\begin{aligned}
& \left(\sum_{k \in \mathbf{Z}} \varphi\left(x_{k}\right)^{r / q} V\left(x_{k}\right)^{-(r / p)}\right)^{1 / r} \\
& \quad+\left(\sum_{k \in \mathbf{Z}}\left(\sup _{\boldsymbol{x}_{k-1}<t<x_{k}}\left(U(t)-U\left(x_{k-1}\right)\right)^{r} V(t)^{-r / p}\right) \frac{\varphi\left(x_{k}\right)^{r / q}}{U\left(x_{k}\right)^{r}}\right)^{1 r}<\infty .
\end{aligned}
$$

Therefore, in view of Theorem 2.1, Lemma 2.5 and Lemma 2.7, we have that

$$
\begin{aligned}
A_{2} & \lesssim\left(\sum_{k \in \mathbf{Z}}\left(\sup _{x_{k-1}<t<x_{k}} U(t)^{r} V(t)^{-r / p}\right) \frac{\varphi\left(x_{k}\right)^{r / q}}{U\left(x_{k}\right)^{r}}\right)^{1 / r} \\
& \lesssim\left(\sum_{k \in \mathbf{Z}} \varphi\left(x_{k}\right)^{r / q} V\left(x_{k}\right)^{-r / p}\right)^{1 / r} \\
& \quad+\left(\sum_{k \in \mathbf{Z}}\left(\sup _{x_{k-1}<t<x_{k}}\left(U(t)-U\left(x_{k-1}\right)\right)^{r} V(t)^{-r / p}\right) \frac{\varphi\left(x_{k}\right)^{r / q}}{U\left(x_{k}\right)^{r}}\right)^{1 / r} \\
& <\infty .
\end{aligned}
$$

Let $1<p<\infty$. For $k \in \mathbb{Z}$, let $h_{k}$ be functions that satisfy the Hardy inequality (2.3) and the Hölder inequality (2.4), that is, functions $h_{k}$ satisfying

$$
\operatorname{supp} h_{k} \subset\left[x_{k-1}, x_{k}\right], \quad \int_{x_{k-1}}^{x_{k}} h_{k}(s) V(s) d s=1,
$$




$$
\begin{gathered}
\left(\int_{x_{k-1}}^{x_{k}}\left(\int_{s}^{x_{k}} h_{k}(t) d t\right)^{1 / p} u(s) d s\right)^{p} \gtrsim\left(\int_{x_{k-1}}^{x_{k}}\left(U(t)-U\left(x_{k-1}\right)\right)^{p^{\prime} / p} V(t)^{-\left(p^{\prime} / p\right)} u(t) d t\right)^{p / p^{\prime}} \\
\int_{x_{k-1}}^{x_{k}} h_{k}(s) d s
\end{gathered}
$$

Now we define the test function

$$
h(s)=\sum_{k \in \mathbf{Z}} a_{k} h_{k}(s),
$$

where $\left\{a_{k}\right\}$ is a sequence of positive real numbers. Then, by using this test function in (4.12) we get

$$
\begin{aligned}
\left(\sum_{k \in \mathbf{Z}} a_{k}^{q / p} \frac{\varphi\left(x_{k}\right)}{U\left(x_{k}\right)^{q}}\left(\int_{x_{k-1}}^{x_{k}}\left(U(t)-U\left(x_{k-1}\right)\right)^{p^{\prime} / p} V(t)^{-\left(p^{\prime} / p\right)} u(t) d t\right)^{q / p^{\prime}}\right)^{1 / q} \\
+\left(\sum_{k \in \mathbf{Z}} a_{k}^{q / p} \varphi\left(x_{k-1}\right) V\left(x_{k-1}\right)^{-(q / p)}\right)^{1 / q} \lesssim\left(\sum_{k \in \mathbf{Z}} a_{k}\right)^{1 / p} .
\end{aligned}
$$

Now, by using Proposition 2.1 for the case $1<p<q<\infty$, we find that

$$
\begin{aligned}
\sup _{k \in \mathbf{Z}}\left(\int_{x_{k-1}}^{x_{k}}\left(U(t)-U\left(x_{k-1}\right)\right)^{p^{\prime} / p} V(t)^{-p^{\prime} / p} u(t) d t\right)^{1 / p^{\prime}} & \frac{\varphi\left(x_{k}\right)^{1 / q}}{U\left(x_{k}\right)} \\
& +\sup _{k \in \mathbf{Z}} V\left(x_{k}\right)^{-1 / p} \varphi\left(x_{k}\right)^{1 / q}<\infty .
\end{aligned}
$$

Hence, by Lemma 2.4 and Lemma 2.8 and performing integration by part, we have that

$$
\begin{aligned}
A_{3} & \lesssim \sup _{k \in \mathbf{Z}} \frac{\varphi\left(x_{k}\right)^{1 / q}}{U\left(x_{k}\right)}\left(\int_{x_{k-1}}^{x_{k}} V(t)^{-p^{\prime}} U(t)^{p^{\prime}} v(t) d t\right)^{1 / p^{\prime}}+\sup _{k \in \mathbf{Z}} \varphi\left(x_{k}\right)^{1 / q} V\left(x_{k}\right)^{-(1 / p)} \\
& \lesssim \sup _{k \in \mathbf{Z}} \frac{\varphi\left(x_{k}\right)^{1 / q}}{U\left(x_{k}\right)}\left(\int_{x_{k-1}}^{x_{k}} V(t)^{-p^{\prime}}\left(U(t)-U\left(x_{k-1}\right)\right)^{p^{\prime}} v(t) d t\right)^{1 / p^{\prime}}+\sup _{k \in \mathbf{Z}} \varphi\left(x_{k}\right)^{1 / q} V\left(x_{k}\right)^{-(1 / p)} \\
& \lesssim \sup _{k \in \mathbf{Z}} \frac{\varphi\left(x_{k}\right)^{1 / q}}{U\left(x_{k}\right)}\left(\int_{x_{k-1}}^{x_{k}} V(t)^{-p^{\prime} / p}\left(U(t)-U\left(x_{k-1}\right)\right)^{p^{\prime} / p} u(t) d t\right)^{1 / p^{\prime}} \\
& +\sup _{k \in \mathbf{Z}} \varphi\left(x_{k}\right)^{1 / q} V\left(x_{k}\right)^{-1 / p}<\infty .
\end{aligned}
$$

Let now $1<p<\infty, 0<q<p$. Then, by using (4.16) and Proposition 2.1 we obtain that

$$
\begin{aligned}
\left(\sum_{k \in \mathbf{Z}} \frac{\varphi^{r / q}\left(x_{k}\right)}{U\left(x_{k}\right)^{r}}\left(\int_{x_{k-1}}^{x_{k}} V(t)^{-p^{\prime} / p}\left(U(t)-U\left(x_{k-1}\right)\right)^{p^{\prime} p} u(t) d t\right)^{r / p^{\prime}}\right)^{1 r} & \\
& +\left(\sum_{k \in \mathbf{Z}} \varphi^{r / q}\left(x_{k}\right) V x_{k}^{-r / p}\right)^{1 / r}<\infty
\end{aligned}
$$


Therefore, by using Theorem 2.1 and Lemma 2.6 and integration by part, we get that

$$
\begin{gathered}
A_{4} \lesssim\left(\sum_{k \in \mathbf{Z}} \frac{\varphi\left(x_{k}\right)^{r / q}}{U\left(x_{k}\right)^{r}}\left(\int_{x_{k-1}}^{x_{k}} V(t)^{-p^{\prime}} U(t)^{p^{\prime}} v(t) d t\right)^{r / p^{\prime}}\right)^{1 / r}+\left(\sum_{k \in \mathbf{Z}} \varphi\left(x_{k}\right)^{r / q} V\left(x_{k}\right)^{-(r / p)}\right)^{1 r} \\
\lesssim\left(\sum_{k \in \mathbf{Z}} \frac{\varphi\left(x_{k}\right)^{r / q}}{U\left(x_{k}\right)^{r}}\left(\int_{x_{k-1}}^{x_{k}} V(t)^{-p^{\prime}}\left(U(t)-U\left(x_{k-1}\right)\right)^{p^{\prime}} v(t) d t\right)^{r p^{\prime}}\right)^{1 / r} \\
+\left(\sum_{k \in \mathbf{Z}} \varphi\left(x_{k}\right)^{r / q} V\left(x_{k}\right)^{-r / p}\right)^{1 / r} \\
\lesssim\left(\sum_{k \in \mathbf{Z}} \frac{\varphi\left(x_{k}\right)^{r / q}}{U\left(x_{k}\right)^{r}}\left(\int_{x_{k-1}}^{x_{k}} V(t)^{-p^{\prime} / p}\left(U(t)-U\left(x_{k-1}\right)\right)^{p^{\prime} / p} u(t) d t\right)^{r / p^{\prime}}\right)^{1 / r} \\
+\left(\sum_{k \in \mathbf{Z}} \varphi\left(x_{k}\right)^{r / q} V\left(x_{k}\right)^{-r / p}\right)^{1 / r}<\infty .
\end{gathered}
$$

Let $q=\infty$ and let $\left\{x_{k}\right\}$ be a discretising sequence for $\varphi$ defined by (2.1). Then, according to (4.11) we have that

$$
\begin{aligned}
\sup _{k \in \mathbf{Z}} \frac{\varphi\left(x_{k}\right)}{U\left(x_{k}\right)} \int_{x_{k-1}}^{x_{k}}\left(\int_{s}^{x_{k}} h(z) d z\right)^{1 p} u(s) d s+\sup _{k \in \mathbf{Z}} \varphi\left(x_{k}\right) & \left(\int_{x_{k}}^{x_{k+1}} h(z) d z\right)^{1 p} \\
& \lesssim\left(\sum_{k \in \mathbf{Z}} \int_{x_{k-1}}^{x_{k}} h(t) V(t) d t\right)^{1 / p} .
\end{aligned}
$$

Let $0<p \leqslant 1$. If we use in (4.17) the test function defined by (4.13) we obtain that

$$
\begin{aligned}
\sup _{k \in \mathbf{Z}} a_{k}^{1 / p} \frac{\varphi\left(x_{k}\right)}{U\left(x_{k}\right)} \sup _{x_{k-1}<t<x_{k}}\left(U(t)-U\left(x_{k-1}\right)\right) V(t)^{-1 / p} & \\
& +\sup _{k \in \mathbf{Z}} a_{k}^{1 / p} \varphi\left(x_{k}\right) V\left(x_{k}\right)^{-(1 / p)} \lesssim\left(\sum_{k \in \mathbf{Z}} a_{k}\right)^{1 p} .
\end{aligned}
$$

Therefore, by Proposition 2.1 we have that

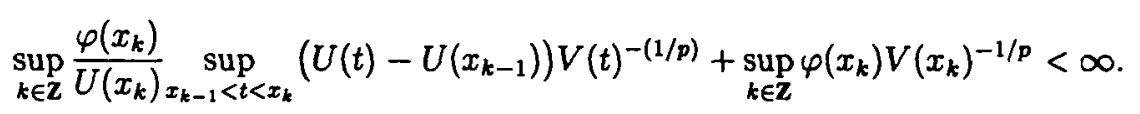

Thus, by Lemma 2.9, we find that

$$
\begin{aligned}
A_{5} & \lesssim \sup _{k \in \mathbf{Z}} \sup _{x_{k-1}<t<x_{k}} V(t)^{-1 / p} \varphi(t) \\
& \lesssim \sup _{k \in \mathbf{Z}} \varphi\left(x_{k}\right) V\left(x_{k}\right)^{-(1 / p)}+\sup _{k \in \mathbf{Z}}\left(\sup _{x_{k-1}<t<x_{k}} U(t) V(t)^{-1 / p}\right) \frac{\varphi\left(x_{k}\right)}{U\left(x_{k}\right)} \\
& \lesssim \sup _{k \in \mathbf{Z}} \varphi\left(x_{k}\right) V\left(x_{k}\right)^{-(1 / p)} \\
& \quad+\sup _{k \in \mathbf{Z}}\left(\sup _{x_{k-1}<t<x_{k}}\left(U(t)-U\left(x_{k-1}\right)\right) V(t)^{-1 / p}\right) \frac{\varphi\left(x_{k}\right)}{U\left(x_{k}\right)}<\infty .
\end{aligned}
$$


Now let $1<p<\infty$. Using in (4.17) the test function defined by (4.15) we obtain that

$$
\begin{aligned}
\sup _{k \in \mathbf{Z}} a_{k}^{1 / p} \frac{\varphi\left(x_{k}\right)}{U\left(x_{k}\right)}\left(\int_{x_{k-1}}^{x_{k}}\left(U(t)-U\left(x_{k-1}\right)\right)^{p^{\prime} p} V(t)^{-\left(p^{\prime} / p\right)} u(t) d t\right)^{1 / p^{\prime}} & \\
& \quad+\sup _{k \in \mathbf{Z}} a_{k}^{1 / p} \varphi\left(x_{k}\right) V\left(x_{k}\right)^{-(1 / p)} \lesssim\left(\sum_{k \in \mathbf{Z}} a_{k}\right)^{1 / p} .
\end{aligned}
$$

Moreover, using Proposition 2.1 we get that

$$
\sup _{k \in \mathbf{Z}}\left(\int_{x_{k-1}}^{x_{k}}\left(U(t)-U\left(x_{k-1}\right)\right)^{p^{\prime} / p} V(t)^{-p^{\prime} / p} u(t) d t\right)^{1 / p^{\prime}} \frac{\varphi\left(x_{k}\right)}{U\left(x_{k}\right)}+\sup _{k \in \mathbf{Z}} V\left(x_{k}\right)^{-1 / p} \varphi\left(x_{k}\right)<\infty .
$$

Hence, by using Lemma 2.4, Lemma 2.8 and performing integration by part, we get that

$$
\begin{aligned}
& A_{6} \lesssim \sup _{k \in \mathbf{Z}} \frac{\varphi\left(x_{k}\right)}{U\left(x_{k}\right)}\left(\int_{x_{k-1}}^{x_{k}} V(t)^{-p^{\prime}} U(t)^{p^{\prime}} v(t) d t\right)^{1 / p^{\prime}}+\sup _{k \in \mathbf{Z}} \varphi\left(x_{k}\right) V\left(x_{k}\right)^{-(1 / p)} \\
& \lesssim \sup _{k \in \mathbf{Z}} \frac{\varphi\left(x_{k}\right)}{U\left(x_{k}\right)}\left(\int_{x_{k-1}}^{x_{k}} V(t)^{-p^{\prime}}\left(U(t)-U\left(x_{k-1}\right)\right)^{p^{\prime}} v(t) d t\right)^{1 / p^{\prime}}+\sup _{k \in \mathbf{Z}} \varphi\left(x_{k}\right) V\left(x_{k}\right)^{-(1 / p)} \\
& \lesssim \sup _{k \in \mathbf{Z}} \frac{\varphi\left(x_{k}\right)}{U\left(x_{k}\right)}\left(\int_{x_{k-1}}^{x_{k}} V(t)^{-\left(p^{\prime} / p\right)}\left(U(t)-U\left(x_{k-1}\right)\right)^{p^{\prime} / p} u(t) d t\right)^{1 / p^{\prime}} \\
&+\sup _{k \in \mathbf{Z}} \varphi\left(x_{k}\right) V\left(x_{k}\right)^{-(1 / p)}<\infty .
\end{aligned}
$$

The corresponding estimates for $A_{7}$ and $A_{8}$ are trivial, by using Lemma 2.1 and taking the test function $f(t)=\underset{0<s<t}{\operatorname{ess} \sup } v(s))^{-1}$. The proof is complete.

Proof of THEOREM 3.2. It is easy to see that

$$
(\chi(0, t))_{u}^{* *}(s) \approx \frac{U(t)}{U(s)+U(t)} .
$$

Using (4.18) we calculate the following norms in Theorem 3.2:

$$
\left\|\left(\chi_{(0, t)}\right)\right\|_{\Gamma_{u}^{q}(w)} \approx\left(\int_{0}^{\infty} \frac{U(t)^{q}}{(U(t)+U(s))^{q}} w(s) d s\right)^{1 / q}
$$

and

$$
\|(\chi(0, t))\|_{\Gamma_{u}^{\infty}(w)} \approx \underset{0<s<\infty}{\operatorname{essup}} \frac{U(t) w(s)}{U(t)+U(s)}
$$

in each of the eight cases considered in Theorem 3.1.

1. For the case $1<p<\infty, 0<p \leqslant q<\infty$, by using (3.3) and (4.19) we find that

$$
\|(\chi(0, t))\|_{\Lambda^{p}(v)_{u}^{\prime}} \approx\|(\chi(0, t))\|_{\Gamma_{u}^{p^{\prime}\left(U(t) p^{\prime} v(t) / V(t) p^{\prime}\right)}}
$$




$$
\approx U(t)\left(\int_{0}^{\infty}\left(\frac{U(s)}{U(t)+U(s)}\right)^{p^{\prime}} \frac{v(s)}{V(s)^{p^{\prime}}} d s\right)^{1 / p^{\prime}}
$$

Thus, using (4.19) and (4.21)

$\sup _{t>0}\left\|\chi_{(0, t)}\right\|_{\Gamma_{u}^{q}(w)}\left\|\chi_{(0, t)}\right\|_{\Lambda^{p}(v)_{u}^{\prime}} U(t)^{-1}$

$$
\begin{aligned}
& \approx \sup _{t>0}\left(\int_{0}^{\infty} \frac{U(t)^{q}}{(U(t)+U(s))^{q}} w(s) d s\right)^{1 q}\left(\int_{0}^{\infty}(U(s) U(t)+U(s))^{p^{\prime}} \frac{v(s)}{V(s)^{p^{\prime}}} d s\right)^{1 / p^{\prime}} \\
& =A_{3} .
\end{aligned}
$$

2. For the case $1<p<\infty, q=\infty$, by using (3.3) and (4.20) we have that $\sup _{t>0}\left\|\chi_{(0, t)}\right\|_{\Gamma_{u}^{\infty}(w)}\left\|\chi_{(0, t)}\right\|_{\Lambda^{p}(v)_{u}^{\prime}} U(t)^{-1}$

$$
\begin{aligned}
& \approx \sup _{t>0}\left(\operatorname{esssup}_{s \in(0, \infty)} \frac{U(t) w(s)}{U(t)+U(s)}\right)\left(\int_{0}^{\infty}\left(\frac{U(s)}{U(t)+U(s)}\right)^{p^{\prime}} \frac{v(s)}{V(s)^{p^{\prime}}} d s\right)^{1 / p^{\prime}} \\
& =A_{6} .
\end{aligned}
$$

For the case $0<p \leqslant 1$, by using (3.3) and (4.18) we obtain that

$$
\left\|\chi_{(0, t)}\right\|_{\Lambda^{p}(v)_{u}^{\prime}} \approx\left\|\chi_{(0, t)}\right\|_{\Gamma_{u}^{\infty}\left(U(t) / V(t)^{1 / p)}\right.} \approx U(t) \operatorname{ess~sup}_{t \in(0, \infty)} \frac{U(s)}{U(s)+U(t)} V(s)^{-1 / p}
$$

3. For the case, $0<p \leqslant 1, p<q<\infty$, by using Lemma 2.2 and (4.22) we get that $\sup _{t>0}\left\|\chi_{(0, t)}\right\|_{\Gamma_{u}^{\infty}(w)}\|\chi(0, t)\|_{\Lambda^{p}(v)_{u}^{\prime}} U(t)^{-1}$

$$
\begin{aligned}
& \approx \sup _{t>0}\left\|\chi_{(0, t)}\right\|_{\Gamma_{i}^{q}(w)}\left\|\chi_{(0, t)}\right\|_{\Gamma_{u}^{\infty}\left(/ U(t) / V(t)^{1 / p)}\right.} U(t)^{-1} \\
& \approx \sup _{t>0}\left(\int_{0}^{\infty}\left(\frac{U(t)}{U(t)+U(s)}\right)^{q} w(s) d s\right)^{1 / q} \operatorname{esssup}_{s \in(0, \infty)} \frac{U(s)}{U(s)+U(t)} V(s)^{-1 / p} \\
& \approx \sup _{t>0}\left(\int_{0}^{\infty}\left(\frac{U(t)}{U(t)+U(s)}\right)^{q} w(s) d s\right)^{1 / q} V(t)^{-1 / p} \\
& =A_{1} \text {. }
\end{aligned}
$$

4. For the case $0<p \leqslant 1, q=\infty$, by using (4.20), (4.22) and Lemma 2.2 we find that

$\sup _{t>0}\|\chi(0, t)\|_{\Gamma_{u}^{\infty}(w)}\|\chi(0, t)\|_{\Lambda^{p}(v)_{u}^{\prime}} U(t)^{-1}$

$$
\approx \sup _{t>0}\|\chi(0, t)\|\left\|_{\Gamma_{u}^{\infty}(w)}\right\| \chi(0, t) \|_{\Gamma_{u}^{\infty}\left((U(t)) /\left(V(t)^{1 / p)}\right.\right.} U(t)^{-1}
$$




$$
\begin{aligned}
& \approx \sup _{t>0} \operatorname{ess} \sup _{s \in(0, \infty)} \frac{U(t) w(s)}{U(s)+U(t)} \operatorname{esssup}_{t \in(0, \infty)} \frac{U(s)}{U(s)+U(t)} V(s)^{-1 / p} \\
& \approx \sup _{t>0}\left(\operatorname{ess}_{s \in(0, \infty)} \frac{U(s) w(s)}{U(s)+U(t)}\right) V(t)^{-1 / p} \\
& =A_{5}
\end{aligned}
$$

5. Now consider the case $1<p<\infty, 0<q<p, r=(p q) /(p-q)$. By using (3.3) (4.19), and (4.21), we have that

$$
\begin{aligned}
& \left(\int_{0}^{\infty}\|(\chi(0, t))\|_{\Gamma_{u}^{q}(w)}^{r-q} w(t) U(t)^{-r}\|\chi(0, t)\|_{\Lambda^{p}(v)_{u}^{\prime}}^{r} d t\right)^{1 / r} \\
& \approx\left(\int_{0}^{\infty}\left\|\left(\chi_{(0, t)}\right)\right\|_{\Gamma_{v}^{q}(w)}^{r-q} w(t) U(t)^{-r}\left\|\chi_{(0, t)}\right\|_{\Gamma_{u}^{p^{\prime}}\left(U(t)^{\left.p^{\prime} v(t) / V(t) p^{\prime}\right)}\right.}^{r^{\prime}} d t\right)^{1 / r} \\
& \approx\left(\int_{0}^{\infty}\left(\int_{0}^{\infty} \frac{U(t)^{q}}{(U(t)+U(s))^{q}} w(s) d s\right)^{(r-q) / q} w(t)\right. \\
& \left.\times\left(\int_{0}^{\infty}\left(\frac{U(t)}{U(s)+U(s)}\right)^{p^{\prime}} \frac{v(s)}{V(s)^{p^{\prime}}} d s\right)^{r / p^{\prime}} d t\right)^{1 / r} \\
& =A_{4} \text {. }
\end{aligned}
$$

6. For the case $0<q<p \leqslant 1$, by using (3.3), (4.19) and (4.22), we obtain that

$$
\begin{aligned}
& \left(\int_{0}^{\infty}\|(\chi(0, t))\|_{\Gamma_{u}^{q}(w)}^{r-q} w(t) U(t)^{-r}\|\chi(0, t)\|_{\Gamma_{u}^{\infty}\left(U(t) / V(t)^{1 / p}\right)}^{r} d t\right)^{1 / r} \\
& \approx\left(\int_{0}^{\infty}\left(\int_{0}^{\infty} \frac{U(t)^{q}}{(U(t)+U(s))^{q}} w(s) d s\right)^{(r-q) / q} w(t) U(t)^{-r}\right. \\
& \left.\quad \times\left(\underset{s \in(0, \infty)}{\operatorname{ess~sup}} \frac{U(t) U(s) V(s)^{-(1 / p)}}{U(t)+U(s)}\right)^{r} d t\right)^{1 / r} \\
& \approx\left(\int_{0}^{\infty}\left(\int_{0}^{\infty} \frac{U(t)^{q}}{(U(t)+U(s))^{q}} w(s) d s\right)^{(r-q) / q} w(t) U(t)^{-r}\left(\sup _{s \in(0, t)} U(s) V(s)^{-(1 / p)}\right)^{r} d t\right)^{1 / r} \\
& =A_{2} .
\end{aligned}
$$

7. For the case $p=q=\infty$, by using (3.3), (4.20) and Lemma 2.2, we have that

$$
\begin{aligned}
& \sup _{t>0}\|(\chi(0, t))\|_{\Gamma_{u}^{\infty}(w)}\left\|\chi_{(0, t)}\right\|_{\Lambda^{\infty}(v)_{u}^{\prime}} \\
& \quad \approx \sup _{t>0}\|\chi(0, t)\|_{\Gamma_{u}^{\infty}(w)}\left\|\left(\chi_{(0, t)}\right)\right\|_{\Lambda^{1}\left(u(\tau) / \text { ess sup } \operatorname{su}_{\ell(0, \tau)} v(s)\right)} U(t)^{-1}
\end{aligned}
$$




$$
\begin{aligned}
& \approx \sup _{t>0}\left(\operatorname{ess~sup}_{s \in(0, \infty)} \frac{U(t) w(s)}{U(t)+U(s)}\right) \frac{1}{U(t)} \int_{0}^{t} \frac{u(\tau)}{{\operatorname{ess~} \sup _{s \in(0, \tau)} v(s)}} d \tau \\
& \approx \operatorname{ess} \sup \frac{w(s)}{U(s)} \int_{0}^{t} \frac{u(\tau)}{\operatorname{ess}_{s \in(0, \infty)} \sup _{s \in(0, \tau)} v(s)} d \tau \\
& =A_{7}
\end{aligned}
$$

8. For the case $0<q<p=\infty$, we have that $r=q$ and by using (3.3), we have that

$$
\begin{aligned}
\left(\int_{0}^{\infty}\left\|\left(\chi_{(0, t)}\right)\right\|_{\Gamma_{u}^{q}(w)}^{r-q} w(t) U(t)^{-r}\left\|\chi_{(0, t)}\right\|_{\Gamma_{u}^{\infty}}^{r}\left(\frac{U(t)}{V(t)^{1 / p}}\right)\right. & d t)^{1 / r} \\
& \approx\left(\int_{0}^{\infty} w(t) U(t)^{-q}\left\|\left(\chi_{(0, t)}\right)\right\|_{\Lambda^{1}\left(u(t) / \text { ess sup }_{s \in(0, t)} v(s)\right)^{q}}^{q} d t\right)^{1 / q} \\
& =\left(\int_{0}^{\infty}\left(\frac{1}{U(t)} \int_{0}^{t} \frac{u(\tau)}{\operatorname{ess~sup}_{s \in(0, \tau)} v(s)} d \tau\right) w(t) d t\right)^{1 / q} \\
& =A_{8} .
\end{aligned}
$$

The proof is complete.

\section{REFERENCES}

[1] M. Ariño and B. Muckenhoupt, 'Maximal functions on classical Lorentz spaces and Hardy's inequality with weights for non-increasing functions', Trans. Amer. Math. Soc. 320 (1990), 727-735.

[2] C. Bennett and R. Sharpley, Interpolation of operators, Pure and Applied Mathematics 129 (Academic Press, Boston, 1988).

[3] M. Carro, A. García del Amo and J. Soria, 'Weak-type weights and normable Lorentz spaces', Proc. Amer. Math. Soc. 124 (1996), 849-857.

[4] M. Carro and J. Soria, 'Weighted Lorentz spaces and the Hardy operator', J. Funct. Anal. 112 (1993), 480-494.

[5] M. Carro and J. Soria, 'Boundedness of some integral operators', Canad. J. Math. 45 (1993), 1155-1166.

[6] M. Carro and J. Soria, 'The Hardy-Littlewood maximal function and weighted Lorentz spaces', J. London Math. Soc. 55 (1997), 146-158.

[7] M. Carro, J. Soria, L. Pick and V.D. Stepanov, 'On embeddings between classical Lorentz spaces', Math. Ineq. Appl. 4 (2001), 397-428.

[8] A. Gogatishvili and L. Pick, 'Duality principles and reduction theorems', Math. Ineq. Appl. 3 (2000), 539-558.

[9] A. Gogatishvili and L. Pick, 'Discretization and anti-discretization of rearrangement-invariant norms', Publ. Mat. 47 (2003), 311-358. 
[10] A. Gogatishvili and L. Pick, 'Embeddings and duality theory for weak clasical Lorents spaces', Canad. Math. Bull. 49 (2006), 82-95.

[11] M.L. Gol'dman, 'Sharp estimates for the norms of Hardy-type operators on the cones of quasimonotone functions', Proc. Steklov Inst. Math. 232 (2001), 1-29.

[12] M.L. Gol'dman, H.P. Heinig and V.D. Stepanov, 'On the principle of duality in Lorentz spaces', Canad. J. Math. 48 (1996), 959-979.

[13] G.G. Lorentz, 'On the theory of spaces $\Lambda^{\prime}$, Pacific J. Math. 1 (1951), 411-429.

[14] B. Opic and A. Kufner, Hardy-type inequalities, Pitman Research Notes in Mathematics (Longman Sci. \& Tech., Harlow, 1990).

[15] E. Sawyer, 'Boundedness of classical operators on classical Lorentz spaces', Studia Math. 96 (1990), 145-158.

[16] G. Sinnamon, 'Spaces defined by level functions and their duals', Studia Math. 111 (1994), 19-52.

[17] G. Sinnamon, 'Embeddings of concave functions and duals of Lorentz spaces', $P u b l$. Mat. 46 (2002), 489-515.

[18] G. Sinnamon, 'Transferring monotonicity in weighted norm inequalities', Collect. Math. 54 (2003), 181-216.

[19] G. Sinnamon and V.D. Stepanov, 'The weighted Hardy inequality: new proofs and the case $p=1$ ', J. London Math. Soc. 54 (1996), 89-101.

[20] V.D. Stepanov, 'The weighted Hardy's inequality for nonincreasing functions', Trans. Amer. Math. Soc. 338 (1993), 173-186.

[21] V.D. Stepanov, 'Integral operators on the cone of monotone functions', J. London Math. Soc. 48 (1993), 465-487.

Mathematical Institute

Academy of Sciences of the Czech Republic

Z̆itná 25

11567 Prague 1

Czech Republic

e-mail: gogatish@math.cas.cz
Department of Mathematics Luleå University of Technology SE-971 87, Luleả

Sweden

e-mail: maria.1.johansson@ltu.se cao@ltu.se

larserik@sm.luth.se

Department of Mathematics

University of Education

P.O. Box 25

Winneba, Ghana

e-mail: okpoticao@yahoo.com cao@ltu.se 